\title{
ENERGIZE: a Phase III study of neoadjuvant chemotherapy alone or with nivolumab with/without linrodostat mesylate for muscle-invasive bladder cancer
}

\author{
Guru Sonpavde ${ }^{*, 1}$, Andrea Necchi ${ }^{2}$, Shilpa Gupta ${ }^{3}$, Gary D Steinberg ${ }^{4}$, Juergen E \\ Gschwend $^{5}$, Michiel Simon Van Der Heijden ${ }^{6}$, Nathalie Garzon ${ }^{7}$, Mohammed Ibrahim \\ Bradley Raybold7, Danny Liaw ${ }^{7}$, Mark Rutstein ${ }^{7}$ \& Matt D Galsky ${ }^{8}$ \\ ${ }^{1}$ Dana-Farber Cancer Institute, Genitourinary Oncology Division, Boston, MA 02215, USA \\ ${ }^{2}$ Fondazione IRCCS Istituto Nazionale dei Tumori, Milan, Italy \\ ${ }^{3}$ Department of Medicine, Masonic Cancer Center, University of Minnesota, Minneapolis, MN 55455, USA \\ ${ }^{4}$ Department of Urology NYU Langone Health, New York University, New York, NY 10017, USA \\ ${ }^{5}$ Department of Urology, Technical University of Munich, Munich, Germany \\ ${ }^{6}$ Department of Medical Oncology, Netherlands Cancer Institute, Antoni van Leeuwenhoek Hospital, Amsterdam, The Netherlands \\ ${ }^{7}$ Bristol-Myers Squibb, Princeton, NJ 08540, USA \\ ${ }^{8}$ Department of Medicine, Icahn School of Medicine at Mount Sinai, Tisch Cancer Institute, New York, NY 10029, USA \\ *Author for correspondence: Tel.: +617 632 2429; Fax: +617 632 2165; gurup_sonpavde@dfci.harvard.edu
}

Immune checkpoint inhibitors have revolutionized the treatment of patients with metastatic urothelial carcinoma. In cisplatin-eligible muscle-invasive bladder cancer (MIBC), cisplatin-based neoadjuvant chemotherapy (NAC) before radical cystectomy improves overall survival. Tumor PD-L1 expression increases in MIBC after NAC, suggesting potential synergy in combining PD1/PD-L1 inhibitors with NAC. IDO1 is overexpressed in bladder cancer and is associated with poor outcomes. Linrodostat mesylate (BMS986205) - a selective, potent, oral IDO1 inhibitor - combined with nivolumab has demonstrated safety and preliminary evidence of clinical activity in metastatic urothelial carcinoma. Here, we discuss the rationale and trial design of the ENERGIZE, a Phase III trial investigating the efficacy of NAC in combination with nivolumab with or without linrodostat followed by postsurgery nivolumab or nivolumab with linrodostat in cisplatin-eligible patients with MIBC. Clinical trial registration number: NCT03661320

First draft submitted: 1 October 2019; Accepted for publication: 27 November 2019; Published online: 11 December 2019

Muscle-invasive bladder cancer (MIBC) is a highly aggressive malignancy characterized by early systemic and distant microscopic dissemination. Locoregional recurrences in the absence of distant recurrences occur in a minority of patients. Prospective Phase III trials have established the use of neoadjuvant cisplatin-based combination chemotherapy preceding radical cystectomy (RC) for resectable (cT2-T4aN0M0) MIBC [1-3]. The landmark SWOG 8710 trial demonstrated that three cycles of neoadjuvant MVAC (methotrexate, vinblastine, doxorubicin and cisplatin) versus RC alone improved 5-year overall survival (OS) significantly (57 vs 43\%; $\mathrm{p}=0.06$ ) [1]. In the international Phase III trial that evaluated neoadjuvant cisplatin, methotrexate and vinblastine, a significant $16 \%$ relative reduction in the risk of death (hazard ratio (HR): $0.84 ; 95 \%$ CI: $0.72-0.99 ; \mathrm{p}=0.037$ ) and an increase in 10 -year survival from 30 to $36 \%$ were observed [2].

The depth of pathological response with neoadjuvant cisplatin-based combination chemotherapy has been shown to be associated with survival; the best outcomes have been seen in patients who attain a pathological complete response (pCR), defined as ypT0 disease [4,5]. A retrospective analysis of the SWOG 8710 trial demonstrated that there appears to be a continuum of long-term survival benefit based on pathological stage after neoadjuvant MVAC: median OS of 13.6, 10.6, 3.7 and 2.4 years associated with pT0, pTa/pT1/pCIS, $\geq \mathrm{pT} 2$ and $\geq \mathrm{pAnyN}$, respectively. However, only approximately $30-35 \%$ of patients achieve pCR translating to improved survival with cisplatin-based combination chemotherapy; therefore, new and more effective therapies are needed [5]. Another potential surrogate end point that robustly correlates with OS is disease-free survival at 2 and 3 years, which is 
compatible with the biology of the disease because the majority of recurrences occur within 2-3 years [6]. Both pCR and disease-free survival are associated with OS at an individual patient level, and the trial-level association remains to be demonstrated but likely differs based on the type of agent.

The therapeutic landscape for metastatic urothelial carcinoma (UC) has been transformed with the approval of multiple PD-1/PD-L1 inhibitors [7]. The PD-L1 inhibitors atezolizumab, avelumab and durvalumab, and PD-1 inhibitors nivolumab and pembrolizumab are approved for patients with disease that progresses after platinumbased therapy [8-12]. Atezolizumab and pembrolizumab are also approved as first-line therapy for cisplatin-ineligible advanced UC with high-PD-L1 expression or platinum-ineligible patients regardless of PD-L1 expression [13,14]. Durable responses are observed in approximately $15-20 \%$ of patients after platinum therapy and in approximately $25 \%$ of patients receiving first-line PD-1/PD-L1 inhibitor therapy [13,14]. PD-1/PD-L1 inhibitors are being combined with platinum-based chemotherapy and CTLA-4-inhibiting immune modulators in randomized Phase III trials evaluating the combination as first-line therapy for metastatic disease, evaluating the combination as firstline therapy for metastatic disease in patients regardless of PD-L1 expression. Indeed, in the settings of metastatic non-small-cell lung cancer and head and neck cancer, the combination of pembrolizumab with platinum-based chemotherapy extended OS regardless of tumor PD-L1 expression [15,16]. The IMvigor130 Phase III trial recently reported an improvement in progression-free survival with the addition of atezolizumab to platinum-based first-line therapy in metastatic UC (stratified HR: 0.82; 95\% CI: 0.70-0.96; $p=0.007$ ) $[17,18]$.

\section{Background \& rationale}

Randomized Phase III trials are already evaluating adjuvant therapy with single-agent PD-1/PD-L1 inhibitors (pembrolizumab, atezolizumab and nivolumab) for high-risk UC with or without neoadjuvant chemotherapy (NAC; NCT03244384, NCT02450331 and NCT02632409, respectively). Moreover, following positive results with adjuvant platinum-based chemotherapy for high-risk upper-tract UC in the POUT trial [19], a Phase III trial is planned to evaluate adjuvant platinum-based chemotherapy with or without a PD-1/PD-L1 inhibitor in this population. Single-agent pembrolizumab and atezolizumab appears feasible, with promising pCR rates (42 and 29\%, respectively), as neoadjuvant therapy for cisplatin-eligible and -ineligible patients with MIBC [20,21]. The PCR rate was associated with high-tumor PD-L1 protein expression by immunohistochemistry and tumor mutational burden. PD-L1 expression is associated with aggressive bladder cancer and has been shown to increase after chemotherapy, suggesting that the PD-1/PD-L1 axis may be a mechanism of resistance to cisplatin-based chemotherapy [22]. Thus, it may be rational to evaluate the combination of PD-1/PD-L1 inhibitors and cisplatinbased NAC.

MVAC remains the regimen supported by Phase III evidence, although gemcitabine plus cisplatin (GC) has been used frequently as NAC in the community setting, based on similar outcomes and better toxicity profiles in the metastatic setting [23,24]. Dose-dense (dd) MVAC (ddMVAC) with granulocyte growth factor support is also feasible and exhibits similar pathological response rates [25,26]. Recently, retrospective studies have yielded conflicting results about the similarity of results from trials involving neoadjuvant GC or MVAC (or ddMVAC), but a randomized Phase II trial recently suggested that they have similar pCR rates [27-31]. There may also be biological reasons to select GC over MVAC/ddMVAC as the backbone of chemoimmunotherapy combinations. In preclinical models, doxorubicin resulted in the expansion of myeloid-derived suppressor cells (MDSCs) [32], and the use of granulocyte growth factors with ddMVAC may also result in the expansion of MDSCs $[32,33]$. In contrast, gemcitabine depletes circulating regulatory T-cell and MDSC counts and reprograms tumor-associated macrophages toward an immunostimulatory M1 phenotype [34]. Furthermore, the shorter duration of ddMVAC treatment may potentially detract from the efficacy of combination with PD-1/PD-L1 inhibitors. In addition, although a creatinine clearance $(\mathrm{CrCl})$ of $\geq 60 \mathrm{ml} / \mathrm{min}$ is typically accepted as a threshold for cisplatin eligibility, split-dose GC (with cisplatin administered on days 1 and 8 every 3 weeks with gemcitabine) has demonstrated feasibility and activity in patients with modest renal dysfunction and an estimated $\mathrm{CrCl}$ of $\geq 40 \mathrm{ml} / \mathrm{min}$ [35,36].

Ongoing Phase I/II trials are evaluating neoadjuvant PD-1 inhibitors in combination with cisplatin-based chemotherapy for cisplatin-eligible MIBC (Table 1). Preliminarily, a Phase I/II trial reported promising activity with the combination of GC and pembrolizumab as neoadjuvant therapy in cisplatin-eligible patients with MIBC, with a pCR rate of $44.4 \%$ and $<\mathrm{pT} 2 \mathrm{~N} 0$ rate of $61.1 \%$ [37]. In contrast with the single-agent pembrolizumab and atezolizumab data, pCR was observed regardless of tumor PD-L1 protein expression with the combination of GC and pembrolizumab in a hypothesis-generating observation. The toxicities of neoadjuvant PD-1/PD-L1 
Table 1. Ongoing Phase I/II trials evaluating neoadjuvant therapy for muscle-invasive bladder cancer.

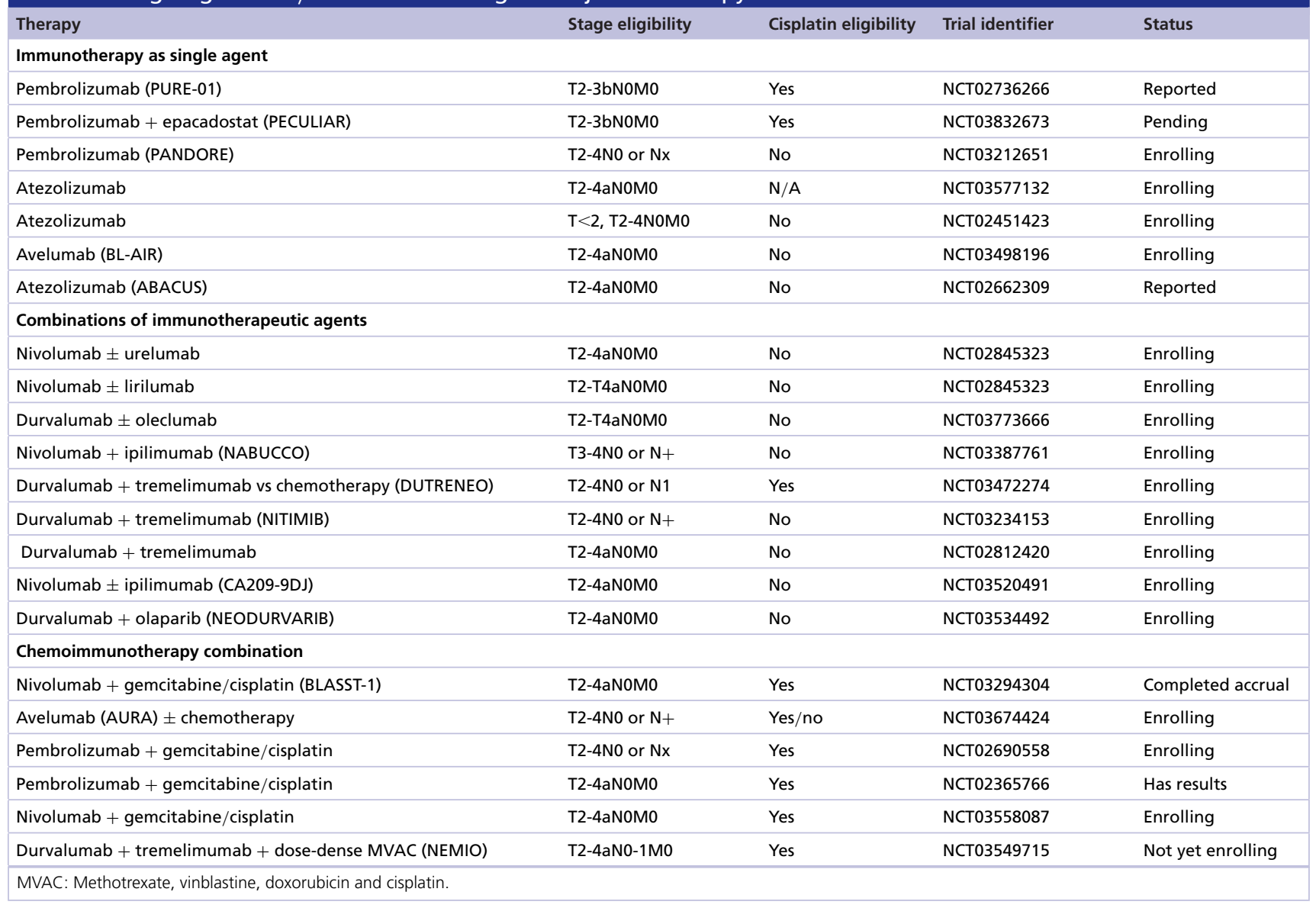

inhibitors alone or in combination with cisplatin-based chemotherapy appear manageable and consistent with previously described toxicities in the metastatic setting. An ongoing Phase II trial (NCT03294304) is investigating the combination of neoadjuvant GC and nivolumab [38].

\section{Linrodostat mesylate (BMS-986205)}

IDO1 is an enzyme that catabolizes the conversion of tryptophan to kynurenine, which reduces immune cell infiltration and upregulates regulatory T-cell counts [39,40]. The expression of IDO1 is higher in multiple malignancies than in normal tissue and is associated with advanced disease and poor clinical outcome [41]. Interestingly, PD1/PD-L1 inhibitor therapy appears to upregulate IDO1 expression in multiple malignancies (Figure 1). Moreover, IDO1 inhibitors appear to enhance the activity of chemotherapy in preclinical models [42]. IDO1 inhibitors have not demonstrated clinical activity as single agents in advanced solid tumors. A Phase III trial demonstrated no additional efficacy with the combination of epacadostat with pembrolizumab in melanoma [43], and conclusive data from other pivotal randomized trials of this combination are not available.

Linrodostat is a selective, potent, once-daily oral IDO1 inhibitor that works early in the IDO1 pathway to reduce kynurenine production from tryptophan [44]. In the metastatic UC setting, the combination of linrodostat with nivolumab in patients with PD-1/PD-L1 inhibitor-naive metastatic UC following one or more prior lines of therapy demonstrated a promising overall response rate of 37\% (three complete responses and seven partial responses in 27 patients), and the disease control rate was 56\% [44]. Treatment-related adverse events (TRAE) occurred in $57 \%$ of patients (grade 3/4,12\%) among 516 patients with any malignancy, and the most common TRAEs were fatigue (15\%) and nausea (12\%). 19 patients (4\%) discontinued due to TRAEs, and three $(<1 \%)$ died from toxicities (myocarditis, Stevens-Johnson syndrome and hepatic failure). The frequency and severity of TRAEs and rate of discontinuation due to TRAEs were lower with the 100- than with the 200-mg linrodostat dose [44]. Therefore, linrodostat $100 \mathrm{mg}$ once daily was considered feasible in combination with nivolumab without 


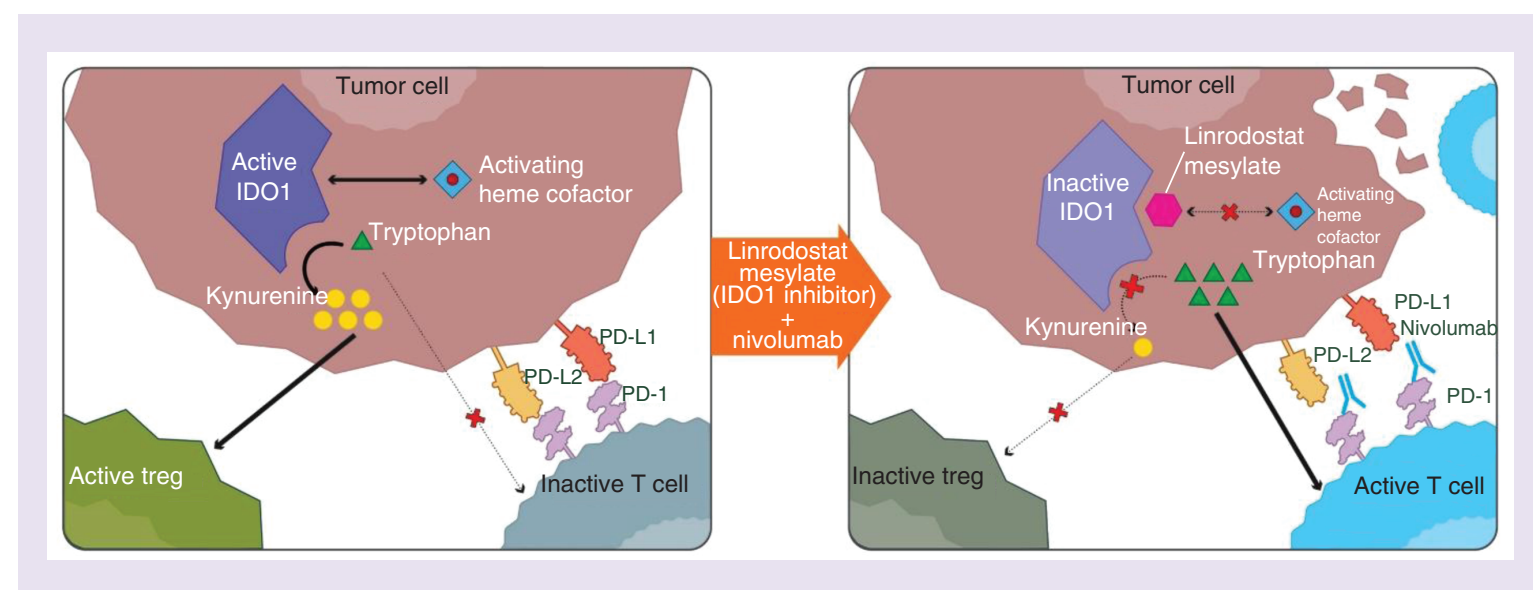

Figure 1. Mechanism of activity of nivolumab and linrodostat. IDO1 expression, which is associated with poor prognosis, allows tumor immune escape through kynurenine production, which stimulates activation of Tregs and suppresses effector T-cell proliferation. Nivolumab is a fully human immunoglobulin G4 PD-1 immune checkpoint inhibitor antibody that binds to the PD-1 receptor and blocks its interaction with PD-L1 and PD-L2. Concurrent IDO1 inhibition and PD-1 pathway blockade has the potential to drive synergistic antitumor effects, reduce kynurenine levels and increase cytotoxic T-cell proliferation within tumors.

Treg: Regulatory T cell.

appearing to exacerbate the toxicities of nivolumab. However, methemoglobinemia was observed - a unique toxicity believed to be related to $p$-chloroaniline production during the metabolism of linrodostat. Notably, no clinically significant methemoglobinemia events were observed at the tested doses. Accrual to the safety lead-in phase of the ENERGIZE Phase III trial, which evaluated the combination of gemcitabine, cisplatin, nivolumab and linrodostat in the neoadjuvant setting, has been completed and safety evaluation is ongoing. Collectively, these data provide a rationale for investigating the combination of cisplatin-based chemotherapy and nivolumab with or without linrodostat $100 \mathrm{mg}$ once daily as neoadjuvant therapy for MIBC.

\section{Methods}

\section{Overall design}

A randomized, partially blind, Phase III trial - ENERGIZE (NCT03661320) - was designed to evaluate and compare the efficacy and safety of neoadjuvant GC alone (Arm A) combined with either nivolumab and linrodostat placebo (Arm B) or nivolumab plus linrodostat (Arm C) followed by RC and postsurgery continuation of immunotherapy in patients with resectable MIBC (Figure 2) [45]. The partial blinding is used to describe that arms $\mathrm{B}$ and $\mathrm{C}$ are placebo-controlled and double-blinded, while the control arm A does not consist of placebo (avoids intravenous placebo, which would be onerous especially in the postoperative component of therapy). The co-primary end points are pCR and event-free survival (EFS). Secondary end points include OS and safety. Although the combination of GC with nivolumab and linrodostat is expected to be tolerable, safety has not been established; therefore, a single lead-in phase will determine the safety of this combination before transition to the randomized Phase III component of the trial. Patients with previously untreated MIBC, a $\mathrm{CrCl}$ of $\geq 50 \mathrm{ml} / \mathrm{min}$, and predominant UC histology and who are eligible for cisplatin-based NAC and RC will be enrolled.

\section{Eligibility criteria}

Cisplatin-eligible patients with UC of the bladder and clinical stage T2-T4aN0M0 are eligible. A predominant $(>50 \%)$ urothelial component is required, and patients must be deemed eligible for $\mathrm{RC}$ by a urologist. Prior Bacillus Calmette-Guérin or other intravesical treatment of nonmuscle invasive bladder cancer (NMIBC) is permitted if completed $\geq 6$ weeks prior to initiating study treatment. An Eastern Cooperative Oncology Group performance status of 0 or 1 and measured or calculated (by Cockroft-Gault formula) $\mathrm{CrCl}$ of $\geq 50 \mathrm{ml} / \mathrm{min}$ are required. Patients are excluded if they are ineligible to receive cisplatin due to comorbidities such as grade $>1$ peripheral neuropathy, hearing loss and New York Heart Association grade $\geq 2$ congestive heart failure. The presence of other conditions that will render patients ineligible include known or suspected autoimmune disease, conditions 


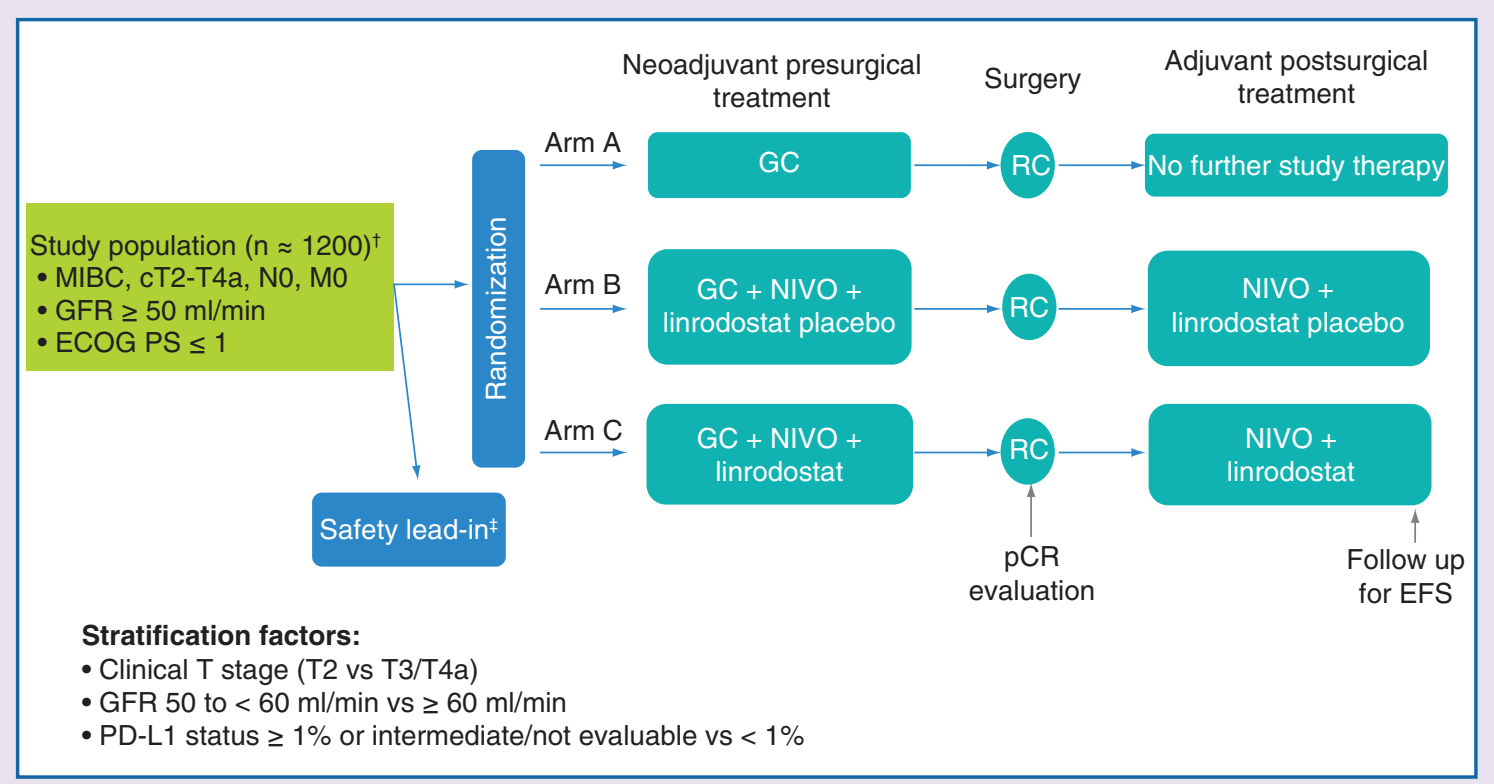

Figure 2. Trial design of ENERGIZE.

$\dagger$ It is anticipated that approximately 1200 participantswill be screened for approximately 954 participants to be randomized overall to the 3 arms in a 1:1:1 ratio.

${ }_{\ddagger}^{\ddagger} \mathrm{A}$ safety lead-in will be conducted prior to randomization to evaluate the tolerability of the proposed arm $\mathrm{C}$ regimen (GC + NIVO + linrodostat).

ECOG PS: Eastern Cooperative Oncology Group performance status; EFS: Event-free survival; GC: Gemcitabine and cisplatin; GFR: Glomerular filtration rate; MIBC: Muscle-invasive bladder cancer; NIVO: Nivolumab; OS: Overall survival; pCR: Pathological complete response; RC: Radical cystectomy.

known to interfere significantly with the absorption of oral medication and any condition requiring systemic corticosteroids (prednisone $>10 \mathrm{mg}$ daily or equivalent) or other immunosuppressive medications within 14 days of randomization. Patients with Type 1 diabetes mellitus, hypothyroidism requiring hormone replacement and skin disorders (such as vitiligo, psoriasis or alopecia) not requiring systemic treatment may enroll. Participants with a personal or family history of cytochrome b5 reductase deficiency or other diseases that predispose them to methemoglobinemia and those with a history of glucose-6-phosphate dehydrogenase deficiency or other congenital or autoimmune hemolytic disorders are excluded. Patients with prior malignancy within the previous 3 years are excluded, except for those with localized curable cancers such as basal or squamous cell skin cancer, prostate cancer with undetectable prostate-specific antigen or carcinoma in situ of the prostate, cervix or breast.

\section{Outcome measures \& planned sample size}

It is anticipated that approximately 1200 participants will be screened for approximately 954 participants to be randomized overall to the 3 arms in a 1:1:1 ratio. The safety lead-in phase will include 18 participants evaluable for the dose-limiting toxicities of the combination of GC, nivolumab and linrodostat. The safe dose is defined as the dose associated with $\leq 33 \%$ of participants exhibiting dose-limiting toxicities. In the randomized part of the trial, the sample size of the Phase III component accounts for the four primary efficacy comparisons: pCR comparison of neoadjuvant nivolumab plus linrodostat plus GC ( $\operatorname{arm~C)}$ versus GC ( $\operatorname{arm~A}$ ), EFS comparison of neoadjuvant nivolumab plus linrodostat plus GC followed by adjuvant nivolumab plus linrodostat following RC $(\operatorname{arm~C})$ versus GC ( $\operatorname{arm~A}), p C R$ comparison of neoadjuvant nivolumab plus GC ( $\operatorname{arm~B}$ ) versus GC $(\operatorname{arm~A})$ and EFS comparison of neoadjuvant nivolumab plus GC followed by adjuvant nivolumab following RC (arm B) versus GC $(\operatorname{arm~A})$. pCR is defined by the proportion of randomized participants with the absence of any cancer (T0, N0 and M0) in pathology specimens after RC. EFS is defined as the time from randomization to progression of disease that precludes surgery, local or distant recurrence, or death due to any cause. The stratification factors for analysis are clinical T stage (T2 vs T3/T4a), $\mathrm{CrCl}$ (50 to $<60$ vs $60 \mathrm{ml} / \mathrm{min}$ ), and tumor PD-L1 status by Dako PD-L1 IHC 28-8 pharmDx assay ( $\geq 1$ vs $<1 \% /$ indeterminate/not evaluable). The secondary objectives of the study are 
Table 2. Ongoing Phase III trials evaluating neoadjuvant therapy for muscle-invasive bladder cancer.

\begin{tabular}{|c|c|c|c|c|}
\hline Trial ID & Sponsor & $\begin{array}{l}\text { Primary end } \\
\text { point(s) }\end{array}$ & Control arm & Experimental arm \\
\hline \multicolumn{5}{|l|}{ Cisplatin eligible } \\
\hline $\begin{array}{l}\text { NCT03661320 } \\
\text { (ENERGIZE) }\end{array}$ & Bristol-Myers Squibb & $\mathrm{pCR}, \mathrm{EFS}$ & GC/split-dose GC & $\begin{array}{l}\text { Control + nivolumab + placebo } \\
\text { Control + nivolumab + linrodostat }\end{array}$ \\
\hline $\begin{array}{l}\text { NCT03732677 } \\
\text { (NIAGARA) }\end{array}$ & AstraZeneca & pCR, EFS & GC/split-dose GC & Control + durvalumab \\
\hline $\begin{array}{l}\text { NCT03924856 } \\
\text { (KEYNOTE-866) }\end{array}$ & Merck & $\begin{array}{l}\text { pCR } \\
\text { EFS }\end{array}$ & $\mathrm{GC}+$ placebo & Control + pembrolizumab \\
\hline \multicolumn{5}{|c|}{ Cisplatin ineligible } \\
\hline $\begin{array}{l}\text { NCT03924895 } \\
\text { (KEYNOTE-905) }\end{array}$ & Merck & $\begin{array}{l}\mathrm{pCR} \\
\mathrm{EFS}\end{array}$ & - & Pembrolizumab \\
\hline
\end{tabular}

EFS: Event-free survival; GC: Gemcitabine and cisplatin; pCR: Pathological complete response.

to evaluate survival and toxicities, evaluate pCR and EFS between arms B and C, compare OS between arms $\mathrm{C}$ and $\mathrm{A}$ and between arms B and $\mathrm{A}$.

Some of the exploratory objectives of the trial are to evaluate pharmacokinetics and time from completion of neoadjuvant therapy to RC, collect tumor tissue for biomarker studies from baseline transurethral resection of bladder tumor and RC tissue and assess patient-reported outcomes. The patient-reported outcome measures include the Functional Assessment of Cancer Therapy (FACT)-General, FACT-Bladder-Cystectomy (FACT-BlCys) and EQ-5D-3L. The FACT-General questionnaire will be used to assess the effects of underlying disease and its treatment on health-related quality of life during treatment. The FACT-Bl-Cys, a validated questionnaire designed for patients undergoing RC, will be administered during neoadjuvant treatment until disease progression or recurrence and at survival follow-up visits. The EQ-5D-3L will be used to assess treatment effects on perceived health status and to generate utility data for health economic evaluations.

\section{Study procedures}

Patients are required to have undergone a transurethral resection of bladder tumor with central pathology review (at baseline and at the time of RC) as well as radiographic imaging at baseline to determine clinical staging and pathology. The baseline tumor also will be assessed for central PD-L1 immunohistochemistry, which is used as a stratification factor. Patients with a baseline $\mathrm{CrCl}$ of $60 \mathrm{ml} / \mathrm{min}$ receive neoadjuvant cisplatin $70 \mathrm{mg} / \mathrm{m}^{2}$ intravenously (iv.) on day 1 every 3 weeks, whereas those with $\mathrm{CrCl}$ of 50 to $<60 \mathrm{ml} / \mathrm{min}$ receive split-dose cisplatin $35 \mathrm{mg} / \mathrm{m}^{2}$ iv. on days 1 and 8 every 3 weeks (one cycle) for four cycles. Gemcitabine is administered at a dose of $1000 \mathrm{mg} / \mathrm{m}^{2}$ iv. on days 1 and 8 every 3 weeks (one neoadjuvant cycle) for four cycles. Patients in arm B receive neoadjuvant GC or split-dose GC and neoadjuvant nivolumab $360 \mathrm{mg}$ iv. on day 1 every 3 weeks for four cycles and placebo oral tablet daily for 12 weeks, followed by adjuvant nivolumab $480 \mathrm{mg}$ iv. every 4 weeks (one adjuvant cycle) for nine cycles and placebo oral tablet daily instituted within 4 months after RC. Patients in arm C receive therapy similar to that in arm B, except that neoadjuvant placebo is replaced by linrodostat (100-mg tablet orally daily) for 12 weeks, followed by adjuvant nivolumab $480 \mathrm{mg}$ iv. every 4 weeks and linrodostat (100-mg tablet orally daily) for nine cycles instituted within 4 months after RC. It is recommended that RC be performed 4-8 weeks after the last dose of neoadjuvant therapy. Laboratory evaluation is performed on days 1 and 8 of every cycle in the neoadjuvant phase and on day 1 of every cycle in the adjuvant phase. RC involves bilateral pelvic lymphadenectomy, including the region below the bifurcation of the common iliac artery and vein, which involves removal of the external and internal iliac and obturator lymph nodes (standard lymphadenectomy). Dissection along the common iliac artery to at least the crossing of the ureter, but most commonly the aortic bifurcation, and sometimes the inferior mesenteric artery is recommended when the surgeon is experienced in performing this extent of surgery - termed an extended lymph node dissection. Radiographic imaging (computed tomography scan/MRI of the chest, abdomen and pelvis) is performed at baseline, before RC, before adjuvant therapy, every 12 weeks for 2 years, and then tapered over the next 3 years.

\section{Conclusion \& future perspective}

ENERGIZE is a pivotal Phase III trial evaluating the value of combining nivolumab or nivolumab plus linrodostat with cisplatin-based combination chemotherapy as neoadjuvant therapy for MIBC, followed by continuation of 
adjuvant immunotherapy. ENERGIZE remains the only planned three-arm Phase III trial and the only trial evaluating an IDO1 inhibitor for this disease. Trials for MIBC sponsored by other entities are also examining the value of combining PD-1/PD-L1 inhibitors with cisplatin-based combination NAC (NCT03732677, NCT03924856; Tables $1 \& 2$ ). In cisplatin-ineligible patients, a chemotherapy-free immune checkpoint inhibitor approach is being examined based on promising Phase II data (NCT03924895; Table 2). The neoadjuvant paradigm to be studied in the ENERGIZE trial (NCT03661320) will provide data about the efficacy, safety, and tolerability of adding a combination of a PD-1 inhibitor and IDO1 inhibitor to cisplatin-based chemotherapy. This study will also inform the development of a precision medicine approach to MIBC through determination of mechanisms of resistance by rapid detection of activity ( $\mathrm{pCR}$ ) and the availability of baseline and post-therapy tumor tissue for molecular analyses.

\section{Executive summary}

\section{Background}

- In cisplatin-eligible muscle-invasive bladder cancer (MIBC), cisplatin-based combination neoadjuvant chemotherapy (NAC) before radical cystectomy (RC) yields pathological complete response ( $p C R$ ) in approximately $30 \%$ of patients, which is robustly associated with improved overall survival.

- PD-L1 expression in tumor tissue increases in MIBC after NAC. IDO1 is overexpressed in bladder cancer and is associated with advanced disease and poor clinical outcomes.

- Linrodostat mesylate (BMS-986205) - a selective, potent, once-daily oral IDO1 inhibitor that reduces kynurenine production - has demonstrated safety and preliminary evidence of clinical activity in combination with nivolumab in metastatic urothelial carcinoma. Nivolumab is a fully human immunoglobulin G4 PD-1 immune checkpoint inhibitor antibody that binds to the PD-1 receptor and blocks its interaction with PD-L1 and PD-L2, programmed death-ligand 2, is approved as therapy for postplatinum advanced UC.

- These data provide a rationale for a Phase III trial, ENERGIZE the efficacy and safety of NAC alone or in combination with nivolumab with or without linrodostat followed by RC and adjuvant immunotherapy in cisplatin-eligible patients with MIBC (NCT03661320).

Methods

- Patients with MIBC (clinical stage T2-T4aNOM0), creatinine clearance of $\geq 50 \mathrm{ml} / \mathrm{min}$ and predominant UC histology, and who are eligible for cisplatin-based NAC and RC are enrolled.

- Participants are randomized to NAC (gemcitabine and cisplatin; arm A), NAC plus nivolumab plus oral placebo (arm B) or NAC plus nivolumab plus linrodostat (arm C) followed by RC. Arms B and C will receive continual nivolumab plus oral placebo or nivolumab plus linrodostat, respectively, following RC.

- Primary end points are $\mathrm{pCR}$ and event-free survival, comparing both arms B and C versus A.

- The secondary objectives of the study are to compare survival, to evaluate safety, and to evaluate $\mathrm{pCR}$, and event-free survival between arms B and C.

- Some of the exploratory objectives of the trial are to evaluate pharmacokinetics and time from completion of neoadjuvant therapy to RC, collect tumor tissue for biomarker studies from baseline transurethral resection of bladder tumor and RC tissue and assess patient-reported outcomes.

Conclusion

- The ENERGIZE trial is the only Phase III trial evaluating an IDO1 inhibitor in cisplatin-eligible MIBC.

- The neoadjuvant paradigm in this study will provide data about the safety, efficacy and tolerability of adding a combination of a PD-1/PD-L1 inhibitor and IDO1 inhibitor to cisplatin-based chemotherapy. This study will also inform the development of a precision medicine approach to MIBC through determination of mechanisms of resistance by rapid detection of activity $(\mathrm{pCR})$ and the availability of baseline and post-therapy tumor tissue for molecular analyses.

Financial \& competing interest disclosure

Guru Sonpavde is a consultant for Bristol-Myers Squibb, Exelixis, Bayer, Sanofi, Pfizer, Novartis, Eisai, Janssen, Amgen, AstraZeneca, Merck, Genentech, EMD Serono and Astellas/Agensys; research support to institution from Bayer, Amgen, Boehringer Ingelheim, Janssen, Merck, Sanofi and Pfizer; author for UpToDate; and speaker for Clinical Care Options, Physicians' Education Resource, Research to Practice, and OncLive. Andrea Necchi is a consultant and advisor for Merck, Incyte, AstraZeneca, Roche, Rainier Therapeutics, Clovis Oncology, Bristol-Myers Squibb, Bayer and Basilea Pharmaceutica; research support to institutio from AstraZeneca, Ipsen, Merck and Incyte; and stock ownership (spouse) in Bayer. Gary D Steinberg is a member of Clinical Trial Protocol Committees for the following companies: Merck, Bristol-Myers Squibb, Janssen and Cold Genesys and scientific advisor/consultant for the following companies: Heat Biologics, Cold Genesys, PhotoCure, Merck, Roche/Genentech, Ciclomed, Taris Biomedical, MDxHealth, Fidia Farmaceuticals, Urogen, Ferring, Aduro, Boston Scientific, Bristol-Myers Squibb, AstraZeneca, Pfizer, Janssen, Epivax Oncology 
and Natera, FKD, Ferring, EnGene Bio and SesenBio. Gary D Steinberg has equity stock/options in Epivax Oncology, Urogen. Michiel Simon Van Der Heijden is a consultation (paid to institution) for AstraZeneca, Bristol-Myers Squibb, Roche/Genentech, Astellas, Seattle Genetics, Merck Sharp \& Dohme and Janssen and grant support (paid to institution) from Bristol-Myers Squibb, Astellas, Roche and AstraZeneca. Nathalie Garzon is an employee of Bristol-Myers Squibb. Mohammed Ibrahim is an employee of Syneos Health outsourced to Bristol-Myers Squibb. Bradley Raybold is an employee of Bristol-Myers Squibb. Danny Liaw has Bristol-Myers Squibb Company Stock. Mark Rutstein is an employee of Bristol-Myers Squibb. Matt D Galsky has received consulting fees from AstraZeneca, Bristol-Myers Squibb, Dragonfly, Genentech, Merck, Numab and Seattle Genetics and has contracted research for AstraZeneca, Bristol-Myers Squibb, Genentech and Merck. The authors have no other relevant affiliations or financial involvement with any organization or entities with a financial interest in or financial conflict with the subject matter or materials discussed in the manuscript apart from those disclosed.

Medical writing support was provided by Kathy Covino, PhD, of Chrysalis Medical Communications, Inc and funded by BristolMyers Squibb Company.

\section{References}

Papers of special note have been highlighted as: $\bullet$ of interest; $\bullet \bullet$ of considerable interest

1. Grossman HB, Natale RB, Tangen CM et al. Neoadjuvant chemotherapy plus cystectomy compared with cystectomy alone for locally advanced bladder cancer. N. Engl. J. Med. 349(9), 859-866 (2003).

-. This is the landmark SWOG 8710 Phase III trial that demonstrated an improvement in overall survival with neoadjuvant methotrexate, vinblastine, doxorubicin and cisplatin (MVAC) chemotherapy for muscle-invasive bladder cancer (MIBC).

2. International Collaboration of Trialists, Medical Research Council Advanced Bladder Cancer Working Party, European Organisation for Research and Treatment of Cancer Genito-Urinary Tract Cancer Group. International Phase III trial assessing neoadjuvant cisplatin, methotrexate, and vinblastine chemotherapy for muscle-invasive bladder cancer: long-term results of the BA06 30894 trial. J. Clin. Oncol. 29(16), 2171-2177 (2011).

- This is the important MRC Phase III trial that demonstrated an improvement in overall and recurrence-free survival with neoadjuvant cisplatin, methotrexate, and vinblastine chemotherapy for MIBC.

3. Advanced Bladder Cancer (ABC) Meta-Analysis Collaboration. Neoadjuvant chemotherapy in invasive bladder cancer: update of a systematic review and meta-analysis of individual patient data advanced bladder cancer (ABC) meta-analysis collaboration. Eur. Urol. 48(2), 202-256 (2005).

- This is a meta-analysis of trials that demonstrated an improvement in overall survival with neoadjuvant cisplatin-based combination chemotherapy for MIBC.

4. Sonpavde G, Goldman BH, Speights VO et al. Quality of pathologic response and surgery correlate with survival for patients with completely resected bladder cancer after neoadjuvant chemotherapy. Cancer 115(18), 4104-4109 (2009).

- This is a retrospective analysis of the SWOG 8710 trial that demonstrated an association between pathological complete response and improvement in survival with neoadjuvant MVAC chemotherapy for MIBC.

5. Petrelli F, Coinu A, Cabiddu M, Ghilardi M, Vavassori I, Barni S. Correlation of pathologic complete response with survival after neoadjuvant chemotherapy in bladder cancer treated with cystectomy: a meta-analysis. Eur. Urol. 65(2), 350-357 (2014).

6. Sonpavde G, Khan MM, Lerner SP et al. Disease-free survival at 2 or 3 years correlates with 5 -year overall survival of patients undergoing radical cystectomy for muscle invasive bladder cancer. J. Urol. 185(2), 456-461 (2011).

- This is a retrospective analysis of a large dataset that demonstrated an association between disease-free survival and survival with or without perioperative chemotherapy for MIBC.

7. Sonpavde G. PD-1 and PD-L1 inhibitors as salvage therapy for urothelial carcinoma. N. Engl. J. Med. 376(11), 1073-1074 (2017).

8. Rosenberg JE, Hoffman-Censits J, Powles T et al. Atezolizumab in patients with locally advanced and metastatic urothelial carcinoma who have progressed following treatment with platinum-based chemotherapy: a single-arm, multicentre, Phase II trial. Lancet 387(10032), 1909-1920 (2016).

9. Patel MR, Ellerton J, Infante JR et al. Avelumab in metastatic urothelial carcinoma after platinum failure (JAVELIN Solid Tumor): pooled results from two expansion cohorts of an open-label, Phase I trial. Lancet Oncol. 19(1), 51-64 (2018).

10. Massard C, Gordon MS, Sharma S et al. Safety and efficacy of durvalumab (MEDI4736), an anti-programmed cell death ligand-1 immune checkpoint inhibitor, in patients with advanced urothelial bladder cancer. J. Clin. Oncol. 34(26), 3119-3125 (2016).

11. Sharma P, Retz M, Siefker-Radtke A et al. Nivolumab in metastatic urothelial carcinoma after platinum therapy (CheckMate 275): a multicentre, single-arm, phase 2trial. Lancet Oncol. 18(3), 312-322 (2017).

12. Bellmunt J, de Wit R, Vaughn DJ et al. Pembrolizumab as second-line therapy for advanced urothelial carcinoma. N. Engl. J. Med. 376(11), 1015-1026 (2017).

13. Balar AV, Galsky MD, Rosenberg JE et al. Atezolizumab as first-line treatment in cisplatin-ineligible patients with locally advanced and metastatic urothelial carcinoma: a single-arm, multicentre, phase 2 trial. Lancet 389(10064), 67-76 (2017). 
14. Balar AV, Castellano D, O'Donnell PH et al. First-line pembrolizumab in cisplatin-ineligible patients with locally advanced and unresectable or metastatic urothelial cancer (KEYNOTE-052): a multicentre, single-arm, phase 2 study. Lancet Oncol. 18(11), 1483-1492 (2017).

15. Gandhi L, Rodriguez-Abreu D, Gadgeel S et al. Pembrolizumab plus chemotherapy in metastatic non-small-cell lung cancer. N. Engl. J. Med. 378(22), 2078-2092 (2018).

16. Rischin D, Harrington KJ, Greil R et al. Protocol-specified final analysis of the phase 3 KEYNOTE-048 trial of pembrolizumab (pembro) as first-line therapy for recurrent/metastatic head and neck squamous cell carcinoma (R/M HNSCC). J. Clin. Oncol. 37(Suppl. 15), [abstract 6000] (2019).

17. Genentech. Genentech's Tecentriq (atezolizumab) plus platinum-based chemotherapy reduced the risk of disease worsening or death in people with previously untreated advanced bladder cancer (2019). www.gene.com/media/press-releases/14801/2019-08-04/genentechs-tecentriq-atezolizumab-plus-p

18. Grande E, Galsky M, Arranz Arija JA et al. IMvigor130: efficacy and safety from a phase 3 study of atezolizumab (atezo) as monotherapy or combined with platinum-based chemotherapy $(\mathrm{PBC})$ vs placebo $+\mathrm{PBC}$ in previously untreated locally advanced or metastatic urothelial carcinoma (mUC). Ann. Oncol. 30(Suppl.5), v851-v934 (2019).

19. Birtle AJ, Chester JD, Jones RJ et al. Results of POUT: a Phase III randomised trial of perioperative chemotherapy versus surveillance in upper tract urothelial cancer (UTUC). J. Clin. Oncol. 36(Suppl.), [abstract 407] (2018).

20. Necchi A, Anichini A, Raggi D et al. Pembrolizumab as neoadjuvant therapy before radical cystectomy in patients with muscle-invasive urothelial bladder carcinoma (PURE-01): an open-label, single-arm, Phase II study. J. Clin. Oncol. 36(34), 3353-3360 (2018).

21. Powles T, Rodriguez-Vida A, Duran I et al. A Phase II study investigating the safety and efficacy of neoadjuvant atezolizumab in muscle invasive bladder cancer (ABACUS). J. Clin. Oncol. 36(Suppl. 15), [abstract 4506] (2018).

- This is a phase II trial of pembrolizumab for MIBC, which demonstrated pathological complete response associated with tumor programmed death-ligand 1 status and tumor mutational burden.

22. McDaniel AS, Alva A, Zhan T et al. Expression of PDL1 (B7-H1) before and after neoadjuvant chemotherapy in urothelial carcinoma. Eur. Urol. Focus 1(3), 265-268 (2016).

- This retrospective analysis demonstrated an upregulation of PDL1 following neoadjuvant cisplatin-based chemotherapy for MIBC.

23. von der Maase H, Sengelov L, Roberts JT et al. Long-term survival results of a randomized trial comparing gemcitabine plus cisplatin, with methotrexate, vinblastine, doxorubicin, plus cisplatin in patients with bladder cancer. J. Clin. Oncol. 23(21), 4602-4608 (2005).

24. Yuh BE, Ruel N, Wilson TG, Vogelzang N, Pal SK. Pooled analysis of clinical outcomes with neoadjuvant cisplatin and gemcitabine chemotherapy for muscle invasive bladder cancer. J. Urol. 189(5), 1682-1686 (2013).

25. Choueiri TK, Jacobus S, Bellmunt J et al. Neoadjuvant dose-dense methotrexate, vinblastine, doxorubicin, and cisplatin with pegfilgrastim support in muscle-invasive urothelial cancer: pathologic, radiologic, and biomarker correlates. J. Clin. Oncol. 32(18), 1889-1894 (2014).

26. Plimack ER, Hoffman-Censits JH, Viterbo R et al. Accelerated methotrexate, vinblastine, doxorubicin, and cisplatin is safe, effective, and efficient neoadjuvant treatment for muscle-invasive bladder cancer: results of a multicenter Phase II study with molecular correlates of response and toxicity. J. Clin. Oncol. 32(18), 1895-1901 (2014).

27. Dash A, Pettus JA IV, Herr HW et al. A role for neoadjuvant gemcitabine plus cisplatin in muscle-invasive urothelial carcinoma of the bladder: a retrospective experience. Cancer 113(9), 2471-2477 (2008).

28. Galsky MD, Pal SK, Chowdhury S et al. Comparative effectiveness of gemcitabine plus cisplatin versus methotrexate, vinblastine, doxorubicin, plus cisplatin as neoadjuvant therapy for muscle-invasive bladder cancer. Cancer 121(15), 2586-2593 (2015).

- This randomized Phase II trial is the only prospective comparison of gemcitabine and cisplatin versus dose-dense MVAC as neoadjuvant therapy for MIBC.

29. Peyton CC, Tang D, Reich RR et al. Downstaging and survival outcomes associated with neoadjuvant chemotherapy regimens among patients treated with cystectomy for muscle-invasive bladder cancer. JAMA Oncol. 4(11), 1535-1542 (2018).

30. Flaig TW, Tangen CM, Daneshmand S et al. SWOG S1314: a randomized Phase II study of co-expression extrapolation (COXEN) with neoadjuvant chemotherapy for localized, muscle-invasive bladder cancer. J. Clin. Oncol. 37(Suppl. 15), [abstract 4506] (2019).

31. Zargar H, Espiritu PN, Fairey AS et al. Multicenter assessment of neoadjuvant chemotherapy for muscle-invasive bladder cancer. Eur. Urol. 67(2), 241-249 (2015).

32. Ding ZC, Lu X, Yu M et al. Immunosuppressive myeloid cells induced by chemotherapy attenuate antitumor CD4+ T-cell responses through the PD-1-PD-L1 axis. Cancer Res. 74(13), 3441-3453 (2014).

33. Kawano M, Mabuchi S, Matsumoto Y et al. The significance of G-CSF expression and myeloid-derived suppressor cells in the chemoresistance of uterine cervical cancer. Sci. Rep. 5, 18217 (2015).

34. Chen C, Chen Z, Chen D, Zhang B, Wang Z, Le H. Suppressive effects of gemcitabine plus cisplatin chemotherapy on regulatory T cells in nonsmall-cell lung cancer. J. Int. Med. Res. 43(2), 180-187 (2015). 
35. Hussain SA, Palmer DH, Lloyd B et al. A study of split-dose cisplatin-based neo-adjuvant chemotherapy in muscle-invasive bladder cancer. Oncol. Lett. 3(4), 855-859 (2012).

36. Galsky MD, Hahn NM, Rosenberg J et al. Treatment of patients with metastatic urothelial cancer "unfit" for cisplatin-based chemotherapy. J. Clin. Oncol. 29(17), 2432-2438 (2011).

37. Hoimes CJ, Albany C, Hoffman-Censits J et al. A phase 1b/2 study of neoadjuvant pembrolizumab (pembro) and chemotherapy for locally advanced urothelial cancer (UC). Ann. Oncol. 29(Suppl.), [abstract LBA33] (2018).

- This Phase II trial reported the activity and safety of gemcitabine and cisplatin combined with pembrolizumab as neoadjuvant therapy for MIBC.

38. BLASST-1 (Bladder Cancer Signal Seeking Trial). Nivolumab, gemcitabine, and cisplatin in treatment of muscle invasive bladder cancer (MIBC) undergoing cystectomy (2019). https://clinicaltrials.gov/ct2/show/NCT03294304

39. Uyttenhove C, Pilotte L, Theate I et al. Evidence for a tumoral immune resistance mechanism based on tryptophan degradation by indoleamine 2,3-dioxygenase. Nat. Med. 9(10), 1269-1274 (2003).

40. Prendergast GC, Malachowski WP, DuHadaway JB, Muller AJ. Discovery of IDO1 inhibitors: from bench to bedside. Cancer Res. 77(24), 6795-6811 (2017)

41. Godin-Ethier J, Hanafi LA, Piccirillo CA, Lapointe R. Indoleamine 2,3-dioxygenase expression in human cancers: clinical and immunologic perspectives. Clin. Cancer Res. 17(22), 6985-6991 (2011).

42. Muller AJ, DuHadaway JB, Donover PS, Sutanto-Ward E, Prendergast GC. Inhibition of indoleamine 2,3-dioxygenase, an immunoregulatory target of the cancer suppression gene Bin1, potentiates cancer chemotherapy. Nat. Med. 11(3), 312-319 (2005).

43. Long GV, Dummer R, Hamid O et al. Epacadostat plus pembrolizumab versus placebo plus pembrolizumab in patients with unresectable or metastatic melanoma (ECHO-301/KEYNOTE-252): a phase 3, randomised, double-blind study. Lancet Oncol. 20(8), 1083-1097 (2019).

44. Luke JJ, Tabernero J, Joshua A et al. BMS-986205, an indoleamine 2, 3-dioxygenase 1 inhibitor (IDO1i), in combination with nivolumab (nivo): updated safety across all tumor cohorts and efficacy in advanced bladder cancer (advBC). J. Clin. Oncol. 37(Suppl. 7), [abstract 4512] (2018).

45. National Institutes of Health. A study of chemo only versus chemo plus nivo with or without BMS-986205, followed by post-surgery therapy with nivo or nivo and BMS-986205 in patients with MIBC (2019). https://clinicaltrials.gov/ct2/show/NCT03661320 